\begin{abstract}
Teresa $\mathrm{Boi}^{*}$
ORCID: 0000-0002-7992-2547

Cagliari, Italia

\section{Pedagogia in Dialogo: ricerca, pratiche e prospettive, Slovenia, Škofja Loka, 14-17 giugno 2018}

Il seminario di studio Pedagogia in Dialogo, una tappa del percorso di approfondimento programmato dall'Inondazione della Pedagogia Educazione-Unità $(\mathrm{EdU})^{1}$, si è proposto di studiare, valorizzare e far conoscere le diverse esperienze educative della Pedagogia di Comunione in atto nei

* Teresa Boi, pedagogista, insegnante, coordinatrice della Commissione internazionale di Educazione-Unità (EdU). Membro del Centro di studi interdisciplinari Scuola Abbà del Movimento dei Focolari. Dottoranda all'Istituto Universitario Sophia a Loppiano in Italia. Address: Via Piave, 15. 00046 Grottaferrata (Rm), Italia; e-mail: pedagogia@focolare.org.

${ }^{1}$ Educazione-Unità (EdU), una rete formata da professionisti del mondo dell'educazione e della formazione, intende promuovere una riflessione e un lavoro di ricerca, per delineare una teoria dell'educazione che ha nella spiritualità di comunione di Chiara Lubich la sua cornice valoriale. Fin dal suo nascere, la spiritualità di comunione si è rivelata portatrice di un rinnovamento non solo spirituale, ma anche sociale e culturale. Lo attestano le numerose opere sociali e le lauree honoris causa assegnate a Chiara Lubich in varie discipline. Anche la nascita di EdU è legata a uno di questi eventi: la laurea honoris causa in pedagogia conferitale dall'Università Cattolica di Washington, il 10 novembre del 2000. In quell'occasione Chiara ha affermato che il Movimento e la sua storia possono essere visti come un grande evento educativo, ché vi sono presenti tutti i fattori dell'educazione ed è pure evidente una teoria dell'educazione che fonda l'agire educativo. EdU può essere definito quindi un laboratorio internazionale per approfondire le linee pedagogiche che emergono dal pensiero e dall'esperienza spirituale di Chiara Lubich e del Movimento dei focolari, in dialogo con l'attuale dibattito sull'educazione, 
luoghi dell'Est Europa. Nella logica della ricerca partecipativa e del sapere esperienziale, è stato approfondito, dai novanta partecipanti provenienti da Croazia, Francia, Italia, Macedonia, Serbia, Slovenia e Spagna ed altri collegati in streaming, il tema del Dialogo quale elemento fondante l'esperienza educativa in contesti multietnici, multiculturali, interreligiosi e globalizzati. Hanno partecipato al seminario insegnanti, educatori, docenti universitari, ricercatori, laureandi e dottorandi.

Caratterizzato per un approccio partecipativo alla ricerca, il seminario si è proposto di favorire la reciprocità tra il sapere teorico, prodotto dai ricercatori, e il sapere esperienziale, frutto della pratica quotidiana di educatori, insegnanti ed operatori. Un'opportunità per favorire lo scambio di riflessioni su metodi, finalità e risultati educativi.

La scuola è prima di tutto una comunità di pratiche educative. L'educare è un'azione performativa che si realizza in diverse forme: quando si spiega, si assegna un compito, si valuta, si esercita la volontà, si dà forma alle proprie idee. Questo fare è un fare su cui si può riflettere, che si può raccontare e la cui condivisione consente la riproducibilità in altri contesti. Per questa finalità, i ricercatori hanno elaborato e inviato alle scuole un call for papers con lo scopo di trasferire alcuni strumenti di ricerca utili agli educatori ed operatori delle scuole per analizzare e poter condividere la situazione in cui operano, per leggerne in modo adeguato le dinamiche e le relazioni.

Nella prima giornata, i partecipanti si sono immersi nella realtà della Scuola materna "Soncni zarek" (Raggio di sole), presso la cittadina slovena di Stara Loka per condividerne l'esperienza durante le ore di attività didattica nelle classi e per conoscere le storie professionali e di vita degli operatori della scuola.

Frequentata da oltre cento bambini suddivisi in tre sezioni di asilo nido e tre di scuola materna (dai 3 ai 6 anni), la scuola è nata nel 2003, in un contesto dove parole come educazione, amore, famiglia, valori sono completamente esclusi dai progetti educativi, con il desiderio e la passione di educare persone nuove con cuore e mente aperti ai più vicini e al mondo intero. Al centro dell'attività educativa è il bambino-persona e il suo fondamentale bisogno di essere rispettato e riconosciuto come un essere unico e irripetibile, dono per gli altri al di là di ogni diversità. Ogni progetto educativo trasmette valori come la pace, la giustizia, la non violenza, l'amministrazione, la

per favorire uno scambio di riflessioni su metodi, finalità e risultati educativi presentati poi in congressi, seminari e convegni in varie parti del mondo. 
cultura, l'accettazione del diverso, ma in particolare a fondamento di ogni azione è posta la relazione interpersonale che ha nell'amore il valore più alto, espressione di quell'arte di amare così come proposta nella spiritualità di Chiara Lubich.

Il dialogo con gli operatori ha permesso di condividerne motivazioni profonde e impressioni. Oltre al titolo di studio, quasi tutti hanno in più qualche specializzazione in musica, arte, biologia, chimica, scienze della comunicazione, lingue slave, sport... e questo arricchisce la diversità dei progetti educativi proposti e aumenta la possibilità di connessioni interdisciplinari. Tangibili l'atmosfera di armonia e di intesa profonda che regna nella scuola, quelle condizioni di comunione, quel sentirsi a proprio agio degli operatori, frutto del vivere positivamente i rapporti e la propria condizione lavorativa.

Il profilo di educatore delineato è stato quello di chi ha trovato nel clima di comunione e nello stile di lavoro della scuola un ambiente favorevole alla piena realizzazione personale e professionale, che qualifica le relazioni interpersonali ed è luogo favorevole per lo sviluppo armonico delle potenzialità che ogni bambino porta con sé. Si è potuto osservare come il funzionamento della realtà scolastica visitata dipende strettamente dal dialogo tra i diversi operatori e dalle relazioni che si vivono tra i vari componenti della comunità educante. Una comunità educante che comprende tutti. Tutti sono educatori e tutti vivono la propria responsabilità ed intenzionalità educativa: insegnanti e genitori, assistenti e cuochi, amministratori. Una comunità educante capace di coinvolgere in un dialogo efficace anche il territorio: una città non particolarmente avvezza alle relazioni interpersonali.

Il tema del seminario "Per una Pedagogia del dialogo" è stato curato da Giuseppe Milan, che ha sottolineato come il dialogo sia un tema di estrema importanza in generale, ma soprattutto per chi opera nel campo dell'educazione. Partendo dalla storia e dai simboli della Slovenia, ha declinato magistralmente il significato del Dialogo nell'ambito della pedagogia, offrendo ricchi spunti teorici e pratici: il dialogo è il modo di essere di noi persone umane, ci qualifica, ci definisce proprio come relazionalità. E Dialogo, etimologicamente, significa parola che si muove tra, parola che mette in relazione, che crea tessuto comunicativo, che permette anche di progettare insieme. La scuola, diceva Milan, è il luogo in cui si realizza questa relazionalità, questa progettualità. Ricerca, desiderio, responsabilità, sosta, progetto, condivisione, perdono, umiltà: sono soltanto alcuni dei pilastri che costituiscono il dialogo. Perché il dialogo è ricerca: cercare l'altro, vederlo in modo nuovo, ri-conoscerlo (conoscerlo ancora); il dialogo è desiderio: rimanda ad una chiamata dall'alto; il dialogo è sosta nell'attenzione profonda all'altro; 
il dialogo è responsabilità; il dialogo è progetto e condivisione; il dialogo è perdono: ricominciare ogni volta ad essere dono; il dialogo è umiltà .

$\mathrm{Su}$ questi temi (pedagogia del dialogo), ha affermato Milan, abbiamo ricevuto, e ancora riceviamo, un grande insegnamento da Chiara Lubich, radice prima e fondamentale ispirazione del nostro parlare, del nostro dialogare, del nostro progettare ed ha concluso citando un brano del testo della laurea honoris causa in pedagogia (Washington, 2000) in cui Chiara Lubich sottolinea che proprio il dialogo è elemento essenziale che dà significato all'agire pedagogico e consente di costruire tra noi un mondo unito:

Nonostante le innumerevoli tensioni del mondo contemporaneo, il nostro pianeta, quasi paradossalmente, tende all'unità: l'unità è un segno e un bisogno dei tempi $[\ldots]$ è perciò implicata, su tutti i piani dell'agire umano, un'azione educativa coerente con le esigenze dell'unità [...]. Dobbiamo perciò realizzare tra noi (continua Lubich) la socialità più autentica, dove si attua una sintesi meravigliosa tra l'istanza pedagogica dell'educazione dell'individuo e l'istanza pedagogica della costruzione della comunità. [...] Naturalmente la nostra esperienza di vita comunitaria si fonda sull'invito di Gesù: "Amatevi come io vi ho amati... Siate una sola cosa": motivazione questa che è di natura religiosa, ma sono straordinari gli effetti sul piano educativo. La finalità da sempre assegnata all'educazione (formare l'uomo, la sua autonomia) si esplica, quasi paradossalmente, nel formare l'uomo-relazione² ${ }^{2}$.

Dalla prospettiva teorica del dialogo si è passati al dialogo tra le cinque scuole di quattro diversi Paesi dell'Est-Europa (Croazia, Slovenia, Macedonia, Serbia) che, per la prima volta, si sono incontrate tutte insieme per condividere la propria esperienza pedagogica.

In rilievo: l'unicità del contributo di ciascuna esperienza educativa nel contesto locale, legata all'incarnazione della stessa radice pedagogica, in risposta ai particolari bisogni del contesto storico, sociale e culturale. Ogni scuola ha potuto dire qualcosa su specifici aspetti, quali la motivazione profonda che la anima, le scelte che accomunano chi lavora nella scuola, il clima relazionale che si vive, i cardini dell'insegnamento, le sfide che restano da affrontare. I temi sono stati tanti, l'esperienza è stata ricca ed arricchente.

${ }^{2}$ Chiara Lubich, "Laurea honoris causa in Pedagogia", Washington D.C., 10 Novembre 2000, in: Chiara Lubich, Una cultura nuova per una società nuova (Roma: Città Nuova, 2002), 171-172. 
Un altro momento fondamentale è stato il dialogo tra accademici: Giuseppe Milan e Michele De Beni, che da diversi anni seguono queste scuole, hanno tessuto come in un prezioso ricamo il filo d'oro dei principi fondanti le esperienze educative: sviluppare la vita interiore del bambino è prioritario rispetto alla pretesa che l'educazione sia finalizzata a realizzare prodotti. Il dialogo, secondo questi ricercatori, è punto qualificante nella relazione simmetrica tra educatore ed educando, nella formazione dell'identità, del pensare e riflettere, del guardare ai propri bisogni e sogni. È lo sviluppo di quello che l'UNESCO nel 1972 ha definito "il capitale invisibile". La crisi educativa sta proprio nel non riconoscere e rendere visibile questo capitale. Lo sviluppo del dialogo interiore porta a scavare nell'umanità che c'è in ciascuno e scoprire il noi dinamico che consente di riconoscere l'alterità e sviluppare uno sguardo creativo capace di essere aperti verso il mondo. Lo sviluppo di questa interiorità è un'azione che non si fa da soli. È un gioco dialogico che educa alla responsabilità e alla libertà nella scoperta del fare. L'educazione dialogica implica lo sviluppo della mente, del cuore e delle mani in un gioco globale dell'arte integrale dell'educare.

C'è però - hanno sottolineato - un Maestro al quale tutti guardiamo ed è a fondamento della nostra educabilità e di ogni progetto educativo: il Maestro tra noi. Spesso il Maestro si cela nel bambino più vulnerabile, nel disabile, nell'immigrato e nello straniero. Una vulnerabilità che richiede ospitalità e dialogo nella complessità della convivenza umana, una convivenza realizzata in prospettiva strumentale ma soprattutto ontologica.

Nel momento conclusivo, riservato al dialogo in plenaria, sono emerse, per gli operatori delle scuole, anche alcune criticità e la difficoltà a sistematizzare adeguatamente l'esperienza educativa in progetti coerenti con la novità della proposta pedagogica che nasce dalla spiritualità di comunione di Chiara Lubich.

Tra domande e proposte si è evidenziato quanto sia importante rinsaldare il dialogo, il rapporto e la condivisione delle varie esperienze educative, in modo da consentire la visibilità della Pedagogia di Comunione sperimentata all'interno delle singole scuole.

Nella prospettiva di trovare una forma di scientificità applicata all'insegnamento, Michele De Beni ha proposto una griglia di osservazione per poter costruire una cultura della documentazione delle pratiche, fatta di processi e risultati riproducibili.

Un'occasione - per le équipes educative che operano nelle diverse scuole - per potenziare, nella prospettiva della Pedagogia di Comunione, le proprie modalità di lettura e analisi delle attività educative, per considerare da 
nuovi punti di vista la complessità della realtà culturale e sociale individuandone sia i punti di forza sia gli elementi di criticità.

Il seminario si è concluso dirigendo lo sguardo dei partecipanti verso un orizzonte condiviso: è in una pedagogia in dialogo, frutto dell'amore scambievole, che l'educazione potrà trovare il suo vero essere. Essa sgorgherà dalla scuola di Gesù Maestro, la scuola per eccellenza dove si impara l'amore quale primaria necessità non solo affettiva, ma anche culturale, di crescita e di progresso sociale. 